\title{
Die Entwicklung der dermatologischen Zeitschriften von 1946 bis 2004
}

A. Scholz

\author{
The Development of Journals for Dermatology from 1946 to 2004
}

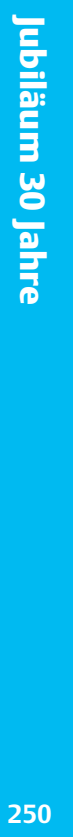

\section{Zusammenfassung}

Am Ende des 2. Weltkrieges war die Produktion aller dermatologischer Zeitschriften eingestellt worden. In den Jahren von 1946 bis 1950 starteten sowohl die traditionellen Blätter wie „Archiv..." und „Dermatologische Wochenschrift“ als auch neu gegründete dermatologische Zeitschriften. In den 60er- und 70erJahren bildete sich eine stärkere Differenzierung zwischen wissenschaftlichen Journalen und dermatologischer Fortbildungsliteratur heraus. In den folgenden Jahrzehnten fusionierten verschiedene Blätter oder mussten ihr Erscheinen einstellen. Viele der in Deutschland gegründeten Zeitschriften publizieren heute vollständig oder partiell in englischer Sprache.

\section{Abstract}

The edition of all dermatological journals was finished at the end of World War II. Both traditional journals as „Archiv... “ and „Dermatologische Wochenschrift" and new founded journals started their production during the years from 1946 to 1950. The differentiation between journals concerning scientific research and clinical education for dermatologists was developed in the 60's and 70's. In the following two decades the production of some journals was either stopped or merged to new journals. Nowadays many German journals are published partially or completely in English language.
Die Eröffnung selbstständiger Kliniken und Abteilungen für Hautkrankheiten, die Gründung einer Fachgesellschaft und die Edition eigener Fachzeitschriften kennzeichneten die Gründungsphase der Dermatologie vor und nach 1900. Die Ernennung der Klinikdirektoren zu Lehrstuhlinhabern mit ordentlichen Professuren und die Einführung des Faches Dermatologie als Pflichtprüfungsfach nach dem 1 . Weltkrieg vollendeten diesen Prozess. Da die deutschen dermatologischen Fachzeitschriften international zu den ältesten und für die Fachgeschichte bedeutendsten Journalen zählen, ergibt sich die spannende Frage, was aus diesen, mit hoher Tradition beladenen Organen geworden ist? Außerdem stellt sich die Frage, in welchem Ausmaß politische und wirtschaftliche Bedingungen Profil und Ausstrahlung der Fachliteratur beeinflusst haben?

\section{Abstieg und Neubeginn}

Die Vertreibung der jüdischen und aus dem Judentum stammenden deutschen Dermatologen und die militärische Dienstverpflichtung der Mediziner nach dem Beginn des 2. Weltkrieges hatte $\mathrm{zu}$ einer nachhaltigen Reduktion der wissenschaftlichen Aktivitäten geführt. Die Anzahl der Artikel in den führenden Zeitschriften hatte sich von 150 bis 200 Aufsätzen im Jahr 1933 auf 10 bis 20 Artikel im Jahr 1944 reduziert. Der Anteil der ausländischen Autoren nahm als Zeichen der internationalen Isolation Deutschlands von Jahr zu Jahr ab. Die Auslieferung der Zeitschriften „Archiv für Dermatologie und Syphilis“ und „Dermatologische Wochenschrift“" wurde 1944 eingestellt [1]. Ein Verlust in der deutschen dermatologischen Zeitschriftenlandschaft muss erwähnt werden. Die Herausgabe der 1893 von Oskar Lassar gegründeten „Dermatologischen Zeitschrift“ wurde in 
Deutschland 1938 eingestellt, weil Heinz Karger, der Sohn des jüdischen Verlegers und Verlagsgründers Samuel Karger, 1937 in die Schweiz emigrieren musste, um sein Leben und den Verlag zu erhalten. Ab 1939 gab der nunmehr in Basel ansässige KargerVerlag die Zeitschrift „Dermatologica“ heraus, die von 1939 1958 von dem Basler Ordinarius Wilhelm Lutz betreut wurde. In Deutschland war die Zeitschrift in der Zeit des NS-Systems nicht mehr erhältlich.

Dementsprechend bestand in der Nachkriegsperiode ein hoher Bedarf an wissenschaftlicher Fortbildungsliteratur. Das erste neugegründete Journal war die „Zeitschrift für Haut- und Geschlechtskrankheiten und deren Grenzgebiete“, dessen erstes Heft in Berlin am 15. Juli 1946 in der von dem Verleger Eduard Grosse sen. wiedergegründeten Berliner Medizinischen Verlagsanstalt erschien. Hierfür war eine Lizenz der Informationsbehörde der amerikanischen Militärregierung erforderlich, die unter der Zulassungsnummer B 225 abgegeben wurde [2]. Herausgeber war der Dermatologe Erich Langer, der sich während der NSZeit wegen seiner jüdischen Herkunft hatte verstecken müssen und nunmehr Chefarzt der Hautabteilung Berlin-Britz am Städtischen Krankenhaus Neukölln war. Von 1949 bis 1957 war er Direktor der Hautklinik an der 1948 neu gegründeten „Freien Universität“" Berlin. Er leitete die Zeitschrift 11 Jahre lang. Sein Schüler Heinz Grimmer übernahm die Schriftleitung nach dem Tod Erich Langers 1957 und führte die Zeitschrift bis 1980. 1958 wechselte Grimmer von Berlin nach Wiesbaden, wo er die Dermatologische Abteilung der Kliniken der Landeshauptstadt Wiesbaden übernahm. Er erweiterte die Zeitschrift von einem bis dahin eher lokal geprägten Mitteilungsblatt zu einem international anerkannten Journal, für das er zahlreiche namhafte inund ausländische Autoren gewinnen konnte [3]. 1948 starteten die beiden deutschen Zeitschriften-Klassiker „Archiv“ und „Dermatologische Wochenschrift“.

Nachdem das „Archiv für Dermatologie und Syphilis“ mit Band 1851944 sein Erscheinen bei Springer eingestellt hatte, erschien 1948 Band 186 dieser Zeitschrift im gleichen Verlag neu. Die Herausgeber Erich Hoffmann, Georg Alexander Rost und Fritz Hussels verwiesen in ihrem Vorwort darauf, dass die bisher in der Zeitschrift dominierende Forschung aus der Notwendigkeit der Zeit heraus durch Beiträge für die Praxis zu ergänzen sei, um „für die deutsche Dermatologie eine Basis zu schaffen, auf der sie wieder aufbauen und zu alter Höhe emporsteigen kann“ [4]. Die „Dermatologische Wochenschrift“" hatte 1944 mit Band 118 ihr Erscheinen eingestellt und startete 1948 mit Band 119, wiederum im Verlag Johann Ambrosius Barth, Leipzig. Dieser Band umfasste Material der Jahre 1947 und 1948. Die Zeitschrift erarbeitete sich unter den praktisch tätigen Dermatologen einen hohen Beliebtheitsgrad.

Der letzte Band des „Zentralblatt für Haut- und Geschlechtskrankheiten“" war Band 71, der im Januar 1945 erschienen war. Das „Zentralblatt..." begann seine Aussendungen mit Band 72, der 1949 herausgegeben wurde. Eine Neugründung von besonderer Dimension war das Erscheinen der Zeitschrift „Der Hautarzt. Zeitschrift für Dermatologie, Venerologie und verwandte Gebiete“ im Januar 1950. Der Herausgeber Alfred Marchionini formulierte die Ansprüche und das Programm in der Erstausgabe. „Während der Herrschaft der Nationalsozialisten war die ,Der- matologische Zeitschrift ' zur Emigration nach der Schweiz gezwungen gewesen." Um diese Lücke zu schließen, verbanden sich die Herausgeber mit dem Springer-Verlag, um die „praktischen Dermatologen mit den wissenschaftlichen und praktischen Fortschritten bekanntzumachen“. Von Anfang an wollte er „im ,Hautarzt eine europäische Tribüne der praktischen und wissenschaftlichen Dermatologie schaffen", die durch amerikanische Mitarbeit erweitert werden sollte [5]. Übersichtsreferate sollten neue Entwicklungen kritisch erläutern, Originalien praktische Probleme und theoretisch bedeutsame Forschungsergebnisse vorstellen, Kurzberichte anfallende klinische Beobachtungen von Klinikern und Praktikern demonstrieren. Berichte und Briefe an den Herausgeber, Tagungsberichte, Buchbesprechungen, Laudationes, Nekrologe, Tagesgeschichte und Kongresskalender erweiterten das Grundsatzprogramm.

1952 erschien das ,Journal für Medizinische Kosmetik und Sexologie“ in der Berliner Medizinischen Verlagsanstalt. Herausgeber und allein verantwortlicher Chefredakteur war der Arzt Ludwig L. Lenz Kairo, Berlin. Die Redaktion für Deutschland lag in den Händen von H. K. Mayr, Berlin, der die Leitung ab Heft 7, 1952 allein übernahm. Die Aufsätze in der Zeitschrift verbanden primär Informationen über medizinische und chirurgische Kosmetik sowie Sexologie. Mit Heft 2, 1953, gliederte sich die Sexologie ab und die Zeitschrift firmierte bis Heft 5, 1956 ausschließlich unter „Journal für medizinische Kosmetik“. Die 1955 neu gegründete „Deutsche Gesellschaft für die Aesthetische Medizin und ihre Grenzgebiete" nutzte das Blatt unter dem reduzierten Titel „Medizinische Kosmetik“ ab 6, 1957, als Kongressorgan. Als Initiator der Gesellschaft, der Jahreskongresse seit 1955 und der Zeitschrift muss der Düsseldorfer Ordinarius Hans Theodor Schreus herausgestellt werden. Sein Einsatz für eine den ganzen Menschen erfassende „Ästhetische Medizin“ ist ein Spezifikum des versuchten geistigen Neubeginns in den 50er-Jahren. H.-Th. Schreus hatte die Bereitschaft vieler Ärzte erfasst, Reformmodelle aus der Zeit vor und nach dem 1. Weltkrieg, die durch den Nationalsozialismus erstickt waren, aufleben zu lassen. Die von ihm geleitete Zeitschrift nahm 1960 noch einmal eine Namensänderung vor und erschien von 1960 bis 1967 unter dem Titel „Aesthetische Medizin“. Es gehört in die geistige Aufbruchstimmung der 50er-Jahre, dass einem Menschenbild in seiner ästhetischen Ganzheit neue Aufmerksamkeit zugewandt wurde.

Im Jahre 1952 erschien im Verlag Editio Cantor K.G. Aulendorf die Zeitschrift „Dermatologische Gutachten“ mit dem Untertitel „Beiträge zur Praxis der Berufsdermatosen“. Der Herausgeber war Karl-Heinz Schölzke, beratender Dermatologe der Kruppschen Krankenanstalten in Essen. A. Marchionini schrieb das Geleitwort, in welchem hervorgehoben wurde, dass bei der Bekämpfung der Berufsdermatosen und insbesondere der Prophylaxe, eine enge Zusammenarbeit zwischen Hautarzt, Gewerbearzt, Betriebsarzt, Werkleitung, Fachklinik, Spezialinstituten und pharmazeutischer Industrie notwendig ist. Die Zeitschrift wurde ein Jahr später umbenannt und hieß seit 1953 „Berufsdermatosen“.

Am 15. März 1954 erschien eineinhalb Jahre nach der Gründung des Verbandes der niedergelassenen Dermatologen das „Mitteilungsblatt“ dieser Vereinigung, um die notwendige Kommuni- 
kation zwischen dem Vorstand und seinen Mitgliedern zu verbessern.

1955 veröffentlichte der Leipziger Johann Ambrosius Barth Verlag erstmals „Allergie und Asthma“ mit dem Untertitel Zeitschrift für Erforschung, Bekämpfung und Verhütung der allergischen Krankheiten und des Asthmas. Zu den Herausgebern gehörte der Berliner Ordinarius G. A. Rost.

Im Juli 1957 startete eine neue, aufstrebende Subdisziplin mit der Zeitschrift „Mykosen“ unter Erich Langer, Berlin als Herausgeber in der Berliner Medizinischen Verlagsanstalt. Heinz Grimmer, Langers Schüler aus der Berliner Zeit, war Teil des Gründungsteams und Mitherausgeber der Zeitschrift. H. Grimmer war ein führender Vertreter der deutschsprachigen Mykologie. Die Repräsentanz von Dermatologen im Herausgeberkollegium entsprach der Wertschätzung des Arbeitsgebietes in den deutschen Hautkliniken dieser Zeitphase. Der Untertitel „Organ für experimentelle und klinische Mykologie“ kennzeichnete die $\mathrm{Ar}$ beitsrichtung (Tab.1).

Tab.1 Zeitschriften für Dermatologie und Grenzgebiete nach 1945

\begin{tabular}{|c|c|c|}
\hline $\begin{array}{l}\text { Jahr der } \\
\text { Herausgabe }\end{array}$ & Titel der Zeitschrift & Herausgeber \\
\hline 1946 & $\begin{array}{l}\text { Zeitschrift für Haut- und } \\
\text { Geschlechtskrankheiten } \\
\text { und deren Grenzgebiete }\end{array}$ & Erich Langer \\
\hline $1947 / 48$ & $\begin{array}{l}\text { Dermatologische } \\
\text { Wochenschrift }\end{array}$ & $\begin{array}{l}\text { H. Löhe, C. Moncorps, } \\
\text { J. Hämel, J. H. Rille, } \\
\text { H. A. Gottron, W. Schönfeld }\end{array}$ \\
\hline 1948 & $\begin{array}{l}\text { Archiv für Dermatologie und } \\
\text { Syphilis }\end{array}$ & $\begin{array}{l}\text { E. Hoffmann, G. A. Rost, } \\
\text { F. Hussels }\end{array}$ \\
\hline 1949 & $\begin{array}{l}\text { Zentralblatt für Haut- und } \\
\text { Geschlechtskrankheiten }\end{array}$ & G. A. Rost, W. Schönfeld \\
\hline 1950 & Der Hautarzt & Alfred Marchionini \\
\hline 1952 & $\begin{array}{l}\text { Journal für Medizinische } \\
\text { Kosmetik und Sexologie } \\
\text { ab } 1960 \text { Aesthetische Medizin }\end{array}$ & $\begin{array}{l}\text { H. K. Mayr, } \\
\text { H. Th. Schreus }\end{array}$ \\
\hline 1952 & $\begin{array}{l}\text { Dermatologische Gutachten } \\
\text { ab } 1953 \text { Berufsdermatosen }\end{array}$ & K. H. Schölzke \\
\hline 1954 & $\begin{array}{l}\text { Mitteilungsblatt Verband der } \\
\text { niedergelassenen Dermatolo- } \\
\text { gen Deutschlands e. V. }\end{array}$ & $\begin{array}{l}\text { Bonk, Ditges, } \\
\text { Dreiner, Voss }\end{array}$ \\
\hline 1955 & Allergie und Asthma & $\begin{array}{l}\text { D. G. R. Findeisen, } \\
\text { H. Kleinsorge, G. A. Rost }\end{array}$ \\
\hline 1957 & Mykosen & Erich Langer \\
\hline
\end{tabular}

\section{Kontinuität und Ausbau}

In den 60er- und 70er-Jahren stabilisierte sich die deutsche Dermatologie und gliederte sich wieder in die internationale Gemeinschaft ein. Neubauten und Sanierungen von Kliniken, Ausbau von unter dem Dach des Fachgebietes vereinigten Spezialgebieten, Erweiterung der experimentellen Forschungsmöglichkeiten kennzeichneten die Situation. Dementsprechend differenzierten sich die Zeitschriften und Publikationsmöglichkeiten. Die politische Spaltung des Landes bedingte eine weitere Eigendynamik. Das „Archiv“ trug der Entwicklung ansteigender For- schungsaktivitäten Rechnung, indem es ab Band 201 im Jahr 1955 seinen Titel zu „Archiv für klinische und experimentelle

Dermatologie“ erweiterte. Es war gleichzeitig, wie schon in der Vergangenheit, nunmehr auch im Titel „Kongressorgan der Deutschen Dermatologischen Gesellschaft", so dass alle 2 Jahre hier die Kongressberichte der DDG dokumentiert waren.

Da sich die Trennung von Ost und West von Jahrzehnt zu Jahrzehnt verstärkte, sollen einige Worte zur Entwicklung der Dermatologie in der DDR gesagt werden. In der stationären wie der ambulanten hautärztlichen Betreuung wurde die Grundtendenz vom Aufbau eines staatlichen Gesundheitswesens konsequent verwirklicht. Die vorherrschende Form der ambulanten Betreuung waren Polikliniken und staatliche Arztpraxen. Der politisch gewollten Abgrenzungsstrategie entsprechend wurde eine eigene Fachgesellschaft für die DDR gegründet, deren Mitgliedschaft mit dem von der SED geforderten Austritt aus westdeutschen, dermatologischen Fachgesellschaften verbunden war.

Die „Dermatologische Wochenschrift“ endete 1968 und wandelte sich mit Band 155 im Jahr 1969 zur „Dermatologischen Monatsschrift". Sie übernahm damit die Funktion, Kongressorgan der Dermatologischen Gesellschaft der Deutschen Demokratischen Republik zu sein. Die Herausgeberschaft übernahmen Wolfgang Gertler, Heinz Egon Kleine-Natrop und Niels Sönnichsen. Die westdeutschen Herausgeber wurden mit Dank verabschiedet. Die Herausgeber formulierten die Aufgaben: „Die ,Dermatologische Monatsschrift ' dient der Veröffentlichung von Forschungsergebnissen und von Arbeiten und Anregungen für die Praxis aus allen Bereichen der Dermatologie und Venerologie sowie deren Grenzgebiete (u.a. Andrologie, ästhetische Medizin, Phlebologie, Mykologie, Arbeitsdermatologie)“ [6]. Die in der DDR wissenschaftlich tätigen Dermatologen hatten primär in dieser Zeitschrift zu publizieren. Veröffentlichungen in westdeutschen Zeitschriften waren nur mit Genehmigung möglich. Da die Zeitschrift international ungenügend beachtet wurde, sind viele $\mathrm{Ar}$ beiten kaum zur Kenntnis genommen worden. Hinter dieser Politik stand das Bestreben der Parteiführung, die Eigenständigkeit der DDR zu beweisen. Damit ging eine Isolierung der DDR mit ungenügender internationaler Repräsentanz von Forschungsergebnissen einher.

Vor den speziellen Journalen muss noch eine Neugründung auf dem allgemeinen Feld der Dermatologie herausgehoben werden. 1974 wurde im Georg Thieme Verlag die Zeitschrift „Aktuelle Dermatologie“ gegründet. Sie sah sich als ein Fortbildungsmedium für Dermatologen und Allergologen in der Praxis. Dementsprechend definierte sie sich primär als Fortbildungszeitschrift und nicht als wissenschaftliches Journal. Dieser Praxisbezug wurde immer wieder der neuen Situation auf dem Zeitschriftenmarkt angepasst. Da die deutsche Dermatologie unverändert durch eine Vielzahl von zum Mutterfach gehörenden Spezialdisziplinen geprägt wurde, sollen einige Spezialblätter in ihrem Verlauf vorgestellt werden.

Die Zeitschrift „Berufsdermatosen“ erschien seit 1978 unter dem neuen Namen „Dermatosen in Beruf und Umwelt“. Sie repräsentierte epidemiologische und klinische Daten sowie die Forschungsaktivitäten auf einem Gebiet, das durch die Häufigkeit arbeitsbedingter Dermatosen einen hohen sozialpolitischen 
Stellenwert hatte. Das 1954 erstmals herausgegebene „Mitteilungsblatt" des Berufsverbandes weitete seine Ausstrahlung mit der Ausdehnung dieser Vereinigung aus. Die Erweiterung des Berufsverbandes um die nicht niedergelassenen Kollegen führte 1976 zu einer Umbenennung in „Der Deutsche Dermatologe“. Die Herausgeber formulierten „Es sollte auch weiterhin keine dermatologische Fachzeitschrift sein, sondern das alle umfassende Bindeglied zwischen den Mitgliedern". Neben berufspolitischen Nachrichten sollten Berichte über Fortbildungsveranstaltungen abgedruckt werden [7].

\section{Strukturwandel}

Obwohl jede Schablonisierung gefährlich ist, sei sie bei diesem Überblick gewagt. Seit den 80er-Jahren entwickelte sich die Forschung in der deutschen Dermatologie durch die Nutzung neuer Arbeitstechniken, der Offenheit für interdisziplinäres Arbeiten und durch die Internationalisierung zunehmend stürmisch. Deutschland erarbeitete sich durch das hohe Engagement sich ausprägender Zentren eine führende Rolle in diesem Wettbewerb. Die Veränderungen spiegelten sich in der Zeitschriftenlandschaft wider.

Die älteste deutsche Fachzeitschrift änderte mit Band 240 im Jahr 1971 ihren Namen in „Archiv für Dermatologische Forschung - Archives for Dermatological Research“. Die Schriftleitung lag bei den Protagonisten dieser Tendenz: O. Braun-Falco, J. Kimmig, G. W. Korting und R. H. Cormane. Das Herausgeberkollegium, der Beirat und eine Gruppe namhafter Editoren repräsentierten die zunehmende Internationalisierung. Ab 1979 wurde die deutsche Bezeichnung aufgegeben, da die Zeitung ausschließlich englischsprachige Aufsätze publizierte. 1979 erschien Band 264 als „Archives of Dermatological Research“, weiterhin im Springer-Verlag gedruckt. Die Zeitschrift steht heute unter der Verantwortung des Editor in Chief Enno Christophers.

Die 1946 neu gegründete „Zeitschrift für Haut- und Geschlechtskrankheiten und deren Grenzgebiete" hatte sich einen breiten Leserkreis erworben, da sie die praktisch-klinische Fortbildung betonte und nie die Bodenhaftung für die in der Praxis tätigen Dermatologen verlor. 1973, noch unter dem Chefredakteur H. Grimmer erhielt die Zeitschrift den Namen „Zeitschrift für Hautkrankheiten $H+G$ “. 1981 bis 1992 lag die Schriftleitung in den Händen des Kölner Ordinarius Gerd Klaus Steigleder, der Garant für wissenschaftliche Aktualität und Alltagsbezug war. Seit dem Januar 1993 übernahm eine gemeinsame Schriftleitung der Herren Th. Krieg, H. F. Merk und H. Hintner die redaktionelle Arbeit aus den Händen von G. K. Steigleder. Gleichzeitig war es zum Wechsel des Verlages gekommen, da die seit 1946 die Zeitschrift betreuende Berliner Medizinische Verlagsanstalt die Geschäfte an den Blackwell Verlag übergab. 2002 ging die Zeitschrift, weiterhin vom Blackwell Verlag betreut, in das neu gegründete „Journal der Deutschen Dermatologischen Gesellschaft", abgekürzt JDDG, als Organ der DDG über. Der erste Herausgeber, Wolfram Sterry, formulierte als Ziel: „Das JDDG fühlt sich der klinischen Forschung und Lehre auf höchstem Niveau im Bereich der Dermatovenerologie und deren Nebenfächern, wie sie traditionell im deutschen Sprachraum definiert sind, verpflichtet."
Der Lebensweg der "Dermatologischen Wochenschrift“ ist ein klares Beispiel für die Abhängigkeit wissenschaftlicher Zeitschriften von Politik und Ökonomie. In der DDR war die Zeitschrift ab 1969 als Publikationsorgan der DDR-Fachgesellschaft im Titel zu „Dermatologische Monatsschrift“ geändert worden. Nach der deutschen Wiedervereinigung konnte sie sich bis 1993 unter diesem Namen halten, wandelte sich ab Band 180 im Jahr 1994 zur „Zeitschrift für Dermatologie“, die dann wiederum mit Band 185 im Jahr 1999 ihre Existenz aufgeben musste und in die „Aktuelle Dermatologie“ aufgenommen wurde. Der Leitung des Georg Thieme Verlags war im Editorial von Band $25 \mathrm{im}$ Jahr 1999 bewusst, dass eine Zeitschrift mit einer Tradition von 117 Jahren bei der jüngeren Zeitschrift „untergeschlüpft“ sei. In dem „sehr schwierigen Markt“ würden jedoch der jüngeren Zeitschrift größere Chancen eingeräumt [8].

Die 1957 in Deutschland gegründete Zeitschrift „Mykosen“ veränderte Spektrum und Sprache ebenfalls in den 80er-Jahren. Seit 1988 firmiert sie unter dem anglophilen Namen „mycoses“ und ist demgemäß von englischsprachigen Aufsätzen dominiert. Die nunmehr im Blackwell Verlag herausgegebene Zeitschrift ist das offizielle Publikationsorgan der „Deutschsprachigen Mykologischen Gesellschaft e.V.“ und steht unter der Herausgeberschaft deutscher Dermatologen: Hans Christian Korting und Herbert Hof.

Eine gleichartige Entwicklung nahm die 1969 in Deutschland von Carl Schirren gegründete Zeitschrift „Andrologie“. 1974 mutierte sie zu dem latinophilen Titel „andrologia“. Da sie unter dem Namen des Fachgebietes als Zeitschrift eingeführt war, entschloss sich die internationale Organisation dieses Spezialgebietes, die Zeitschrift als offizielles Organ des Comité Internacional De Andrologia zu nutzen. Sie steht heute gedruckt im Blackwell Verlag, unter deutscher Herausgeberschaft von Wolf-Bernhard Schill und Martin Bergmann.

Die aus den „Berufsdermatosen“ hervorgegangene, seit 2000 unter „Dermatologie in Beruf und Umwelt“ firmierende Zeitschrift konnte bis 2002 ihre Eigenständigkeit bewahren. Ab 2003 wurde sie als Beilage in die Zeitschrift „Allergologie“ eingefügt. Ihre Öffnung gegenüber englischsprachigen Beiträgen hatte sie seit 1977 mit dem Zweittitel „Occupational and Environmental Dermatology“ demonstriert.

Aus der Marktwirtschaft muss hier ein Begriff übernommen werden, da er harte Entscheidungen beschönigen soll. Der „Bereinigung“ des Zeitschriftenmarktes diente das Ende des seit $1921 \mathrm{im}$ Springer-Verlag gedruckten „Zentralblatt für Haut- und Geschlechtskrankheiten sowie deren Grenzgebiete“. Die Referate dieser Zeitschrift waren eine Informationsquelle für internationale Artikel auf dem Gebiet der Dermatologie und Venerologie gewesen. Die sich immer mehr ausweitenden elektronischen Medien verdrängten diese „Papierform“, so dass das „Zentralblatt ..." 1996 mit Band 168 sein Erscheinen einstellte.

Das gegenläufige Beispiel ist die marktabhängige Herausgabe neuer Zeitschriften auf dem Gebiet der kosmetischen Medizin, in das die Dermatologie zunehmend involviert ist. An erster Stelle soll die neue Edition der einleitend erwähnten „Ästhetischen Medizin“ erwähnt werden, die mit Band 16 im Jahr 1967 ihr Er- 
scheinen eingestellt hatte. 1996 startete die Berliner Medizinische Verlagsanstalt im Anschluss an die Zählweise der vorangegangenen Zeitschrift mit Band 17 als „Kosmetische Medizin Cosmetic Medicine“. Da sie das Organ der „Vereinigung für Ästhetische Dermatologie und Lasermedizin“ und der „Deutschen Gesellschaft für Ästhetische Dermatologie“ ist, sollte sie in diesem Überblick nicht fehlen. Die Arbeitsgemeinschaft „Ästhetische Dermatologie und Kosmetologie“ gibt seit 2003 ebenfalls eine eigene Zeitschrift mit dem Namen „Ästhetische Dermatologie“ heraus. Eine Neuerung aus dem Jahr 2000 ist das Journal „Zeitschrift für ästhetische und operative Dermatologie“, in der namhafte Dermatologen mit operativer Spezialisierung ihre operationstechnischen Erfahrungen publizieren.

Aus der Breite des Angebots sollen noch zwei Blätter erwähnt werden, die sich der allgemeinen Fortbildung gewidmet und eine längere Erscheinungszeit bewältigt haben. Seit 1989 gibt der Viavital Verlag die Zeitschrift „HAUT“ heraus, die in Excerpta Medica gelistet ist und seit längerer Zeit unter der Schriftleitung von Frau Maria Zabel steht, die mit einem wissenschaftlichen Beirat immer den Praxisbezug im Mittelpunkt ihrer Auswahl sieht.

Die Hamburger OmniMed Verlagsgesellschaft ediert seit 1993 die „derm“, die sich als Forum ärztlicher Fortbildung und Qualitätssicherung versteht. Die Redaktion wird von einem umfassenden Beirat bekannter Dermatologen gestützt.

\section{Zusammenfassung}

Die Vitalität der Dermatologie spiegelte sich in der Wiederbelebung der wissenschaftlichen Veranstaltungen und im Zeitschriftenwesen wider. Das Jahr 1948 kann als das Jahr des Neubeginns bezeichnet werden. Es gründeten sich die Fachgesellschaften nach der erzwungenen Unterbrechung neu und die DDG hielt im Oktober 1949 ihren ersten Kongress nach dem Krieg ab. 1948 nahmen die klassischen Zeitschriften „Archiv“ und „Wochenschrift" ihre Arbeit wieder auf. 1950 folgte als neue Zeitschrift „Der Hautarzt“, dem in den 50er-Jahren weitere Neuerungen für Spezialgebiete folgten. Tendenzen und Schwerpunkte der wissenschaftlichen Dermatologie lassen sich aus Themen in Zeitschriften ableiten. Die Betrachtungsebene hat sich in der Nachkriegszeit weiter von der klinischen Morphologie zur funktionalen Interpretation gewandelt. Die Verlagerung in der Morphologie betraf die durch neue technische und biologische Möglichkeiten erreichbaren Strukturen. In vielen Universitäten wurde die Ultrastruktur der Haut erforscht und in Beiträgen verfeinert. Die von Köln aus sich seit den 50er-Jahren ausbreitende Histochemie brachte ebenso neue Erkenntnisse wie die Biochemie, die später ihre neue Ebene in der Immunologie fand. Hier kamen aus der Dermatologie gerade bei systemischen Erkrankungen entscheidende Beiträge für die Gesamtmedizin.

Der epidemiologisch nachweisbare Wechsel von Krankheitsbildern bestimmte die Forschungsarbeit. Der Rückgang von Pyodermien und Epizoonosen, der Wandel von externen zu systemischen Mykosen, die Zunahme von Allergien und Tumoren prägten die neuen Arbeitsfelder. Der den klinischen Alltag beherrschende Wandel betraf weiterhin die Therapie mit dem Wechsel in der Dominanz von externer zu interner Therapie. Berichte zu den Erfolgen der Antibiotika, verschiedener Chemotherapeutika, der intern eingesetzten Antimykotika und neu eingeführten Nebennierenrindenhormone bestimmten die Inhalte der Veröffentlichungen.

Die prägenden Protagonisten der ersten Nachkriegsphase waren Alfred Marchionini und Oscar Gans auf der einen und Heinrich A. Gottron und Hans-Theodor Schreus auf der anderen Seite. A. Marchionini und O. Gans führten Deutschland in die internationale Gemeinschaft zurück, H. A. Gottron und H.-Th. Schreus waren die Individualisten, die in Deutschland selbst dem Fach neue Anerkennung verschafften. H. A. Gottron betonte immer wieder seine Auffassung der Dermatologie als Querschnittsfach zwischen Innen und Außen. Es war eine Zeit, in der die ganzheitliche Betrachtung des hautkranken Menschen immer wieder eingefordert wurde.

Dem Aufbau des neuen Hauses der Dermatologie in den 50erJahren folgte der Ausbau in den 60er- und 70er-Jahren. Diese Phase ist mit den zwischen 1922 und 1925 geborenen Persönlichkeiten Otto Braun-Falco, Gerd-Klaus Steigleder, Theodor Nasemann und Siegfried Borelli verbunden, die wissenschaftlich und gesundheitspolitisch dem Fachgebiet neue Anerkennung verschafften und mit ihren Schülern die Gegenwart bestimmen. In den Zeitschriften erfolgte eine immer stärkere Differenzierung zwischen Grundlagenforschung und klinisch orientierter Fortbildung. Das „Archiv..." wurde immer mehr zum Sammelbecken der reinen und angewandten Forschung. Die anderen Fachzeitschriften bemühten sich als Bindeglied zwischen Praxis, Klinik und Laboratorium zu wirken. Die Umstrukturierung des dermatologischen Zeitschriftenspektrums hat seit den 80er-Jahren sowohl zu einer Differenzierung als auch zu einem Überangebot geführt. Totales oder schleichendes Verschwinden von Titeln gehörte zu diesem Prozess. Das „Zentralblatt für Haut- und Geschlechtskrankheiten“ gab 1996 auf. Die traditionellen Säulen der Wissensaufbereitung wie „Archiv“ und „Wochenschrift“ sowie die „Zeitschrift für Hautkrankheiten $\mathrm{H}+\mathrm{G}$ “ sind in einer fortlaufenden Transformation bis auf mikroskopische Hinweise in den Nachfolgejournalen verschwunden (Tab. 2). Das entspricht dem Verlust von Traditionsnamen in der deutschen Wirtschaft auf Grund von Fusionen.

Die Dermatologie vollzog eine häufig beklagte, aber im internationalen Wettbewerb unbeeinflussbare Anglisierung von Zeitschriften, die editoriell von deutschen Herausgebern bestimmt werden. Das stärkte die deutsche Position in der immer wieder kritisierten, jedoch überall entscheidenden Bewertung wissenschaftlicher Leistungen mit Impact-Faktoren. Ohne die deutsche Zeitschrift „Archives ...“ würden wissenschaftlich hochkarätige Beiträge deutscher Forscher nur noch in den USA gedruckt. Diese Tendenz realisiert auch das neue Organ der DDG, die Zeitschrift „JDDG“. In diese anglophile Tendenz reiht sich die Internationalisierung der in Deutschland entstandenen Journale ein: „mycosis“, „andrologia“, „Occupational and Environmental Dermatology“.

Dieser Tendenz steht die Neugründung einer Reihe von Informationsblättern gegenüber, die die Praxisnähe suchen, jedoch die schreibende Zunft und die Leser überfordern. Die Folge sind eine Vielzahl von Aufsätzen der gleichen Autoren, die ihren PC 
Tab. 2 Transformation, Fusion und Ende von dermatologischen Zeitschriften

\begin{tabular}{ll}
\hline \multicolumn{1}{c}{ Zeit } & Name der Zeitschrift \\
\hline 1882 & $\begin{array}{l}\text { Monatshefte für Praktische Dermatologie } \\
\text { Dermatologische Wochenschrift }\end{array}$ \\
1912 & Dermatologische Monatsschrift \\
1969 & Dermatologische Zeitschrift \\
1994 & Fusion und Übergang in Aktuelle Dermatologie \\
\hline 1999 & Archiv für Dermatologie und Syphilis \\
1955 & Archiv für klinische und experimentelle Dermatologie \\
1971 & Archiv für dermatologische Forschung \\
1979 & Archives for Dermatological Research \\
\hline 1946 & Zeitschrift für Haut- und Geschlechtskrankheiten \\
1973 & Zeitschrift für Hautkrankheiten H + G \\
2002 & Fusion und Übergang in Journal der Deutschen \\
& Dermatologischen Gesellschaft \\
\hline Gründung 1921 & $\begin{array}{l}\text { Zentralblatt für Haut- und Geschlechtskrankheiten } \\
\text { Ende der Zeitschrift }\end{array}$ \\
\hline Auflösung 1996
\end{tabular}

geschickt nutzen, um durch Zusammensetzen von Blöcken aus verschiedenen, bisher schon vorhandenen Aufsätzen „neue Stücke“ zu produzieren.

Ein bisher unantastbarer Fels in dem wogenden Meer des Papiers ist der nunmehr bei Band 55 angelangte „Hautarzt“, der sein seit der Erstausgabe 1950 begonnenes Profil beibehalten hat. Er verwirklicht das von dem Gründer der Zeitschrift, Alfred Marchionini, formulierte Ziel „die paraktischen Dermatologen mit den wissenschaftlichen und praktischen Fortschritten bekanntzumachen“. Die hohe, gutachterlich kontrollierte Qualität garantiert ihm die Zuneigung einer großen Leserschaft. Die „Aktuelle Dermatologie“ hat sich bis zu ihrem nunmehr 30-jährigen Jubiläum im dermatologischen Zeitschriftenspektrum eine fest umrissene Position erarbeitet.

\section{Literatur}

${ }^{1}$ Scholz S, Scholz A. Die Wissenschaftsentwicklung in der Dermatologie in Deutschland im Verhältnis zur Emigration jüdischer Hautärzte während des Nationalsozialismus. Wien Klin Wochenschr 1998; 110/4-5: 185 - 189

${ }^{2}$ Grosse E. Vom Traum zur Wirklichkeit der Freiheit. Berlin: Berliner Medizinische Verlagsanstalt, 1996

${ }^{3}$ Steigleder GK. Zum Tode von Heinz Grimmer. Z Hautkr 1982; 57: 935

${ }^{4}$ Hoffmann E, Rost GA, Hussels F. Vorwort. Arch Derm Syph 1948; 186: $1-2$

${ }^{5}$ Marchionini A. Dem „Hautarzt“ zum Geleit. Hautarzt 1950; $1: 1$ - 2

${ }^{6}$ Herausgeber und Verlag. Zum Geleit. Dermatol Monatsschr 1969; 155: $1-2$

${ }^{7}$ Schirren C, Steinmeier T. 40 Jahre Berufsverband Deutscher Dermatologen. Hamburg: MEDI-A-DERM Verlag, 1992: 78 - 79

${ }^{8}$ Georg Thieme Verlag. In eigener Sache. Akt Dermatol 1999; 25: 285

\section{Buchbesprechung}

\section{Kosmetische Dermatologie}

W.-I. Worret, W. Gehring (Hrsg)

Heidelberg: Springer, 2004. 386 S., 460 Abb., 77 Tab. Geb.

$€ 119,95$. ISBN 3-540-43071-7

Die kosmetische Dermatologie ist einerseits ein Bestandteil der Dermatologie und andererseits aber auch ein sich eigen entwickeltes Gebiet mit großer Zukunftspotenz und auch mit zunehmenden kommerziellen Möglichkeiten. Dies wird unterstützt durch einen ästhetischen Wertewandel, der getragen wird von einem Bedeutungswandel von der Korrektur zur Prophylaxe, von der Beseitigung von Unerwünschtem zur Betonung des Ästhetischen. Eine zusammenfassende Darstellung ist also notwendig. Sie liegt hier vor und ist gelungen.

Die beiden Dermatologen, Wolf-Ingo Worret aus München und Wolfgang Gehring aus Karlsruhe haben um sich 35 Autoren versammelt und das Werk mit einer vorbildlichen Konstruktion geschaffen. Die meisten der Autoren sind langfristig im Thema engagiert und seit acht Jahren in der Arbeitsgemeinschaft für ästhetische Dermatologie und Kosmetologie der DDG tätig und versammelt. Sie kennen sich also und ergänzen sich bestens. 386 Seiten umfasst das Werk, ist hervorragend mit 200 Abbildungen und 41 Tabellen ausgestattet und vom Verlag bestens begleitet und ausgerüstet.

Zu Beginn ist die Physiologie und die Anatomie der Haut, die Pathologie kosmetischer Störungen und auch die Pflege in gebotener Kürze dargebracht. Die medizinischen und rechtlichen Grundlagen nehmen mehr Platz ein, umfassen sie doch auch die Forschung und die Anwendungsforschung. Erfreulich ist die Einbindung der Fachkollegen aus Industrie und Forschungsinstituten. Ausführliche Kapitel berichten über spezielle Kosmetika, über die operativen Möglichkeiten und wiederum spezielle kosmetische Behandlungsverfahren. Sie sind ausführlich und präzise dargestellt. Ein eigenes Kapitel widmet sich den speziellen Indikationen zur kosmetischen oder ästhetischen Behandlung. Hier werden auch Erfolge, Grenzen, Nebenwirkungen und Schäden aufgeführt, Hoffnung und Realität werden abgegrenzt.

Kosmetik, Kosmetische Dermatologie und ästhetische Anliegen sind bestens vertreten, kompetent dargestellt und hervorragend präsentiert. Auch die Grenzen des Buches sind klar gesetzt. So sind ausgesprochen dermatologische Anliegen ausgeklammert, z.B. die Akne und die Vitiligo.

Das Buch wird ergänzt durch ein Kapitel über die Etablierung und praktischen Führung kosmetischer Institute und ein eigenes Kapitel über die rechtliche Grundlagen des ganzen Arbeitsbereiches.

Der Zugang wird über ein ausführliches Inhaltsverzeichnis am Anfang und über ein hervorragendes Sachverzeichnis am Schluss leicht gehalten. Ein erfreuliches Buch, dem weite Verbreitung gewünscht wird.

E. G. Jung, Heidelberg 